\title{
Effects of ICI 118.551, a selective beta- 2 adrenergic blocking agent on the guinea pig cardiac excitability and ventricular fibrillation threshold
}

\author{
B. Cuparencu, C. Tomus*, Manuela Gozariu**, Alina Cuprian* \\ Department of Pharmacology, University of Oradea, Romania \\ * Department of Pharmacology, University of Medicine and Pharmacy \\ "Iuliu Hațieganu", Cluj-Napoca, Romania \\ ** Laboratoire de Neurophysiologie, Hopital Pitie - Salpetriere, Paris, France
}

Received: May 21, 1999

Accepted: November 30, 1999

\begin{abstract}
In isolated guinea pig perfused hearts ICI 118.551, a selective beta 2 adrenoceptor antagonist, induced transient ventricular extrasystoles. Following the termination of the perfusion, a very significant increase of both the ventricular fibrillation threshold and the refractory periods were measured. In guanethidine pretreated hearts, ICI 118.551 failed to induce premature beats. At the same time the fibrillation threshold and refractory periods exhibited a very significant decrease. The perfusion of equimolecular concentration of metoprolol, a beta-1-adrenoceptor antagonist, and $(+)$ propranolol, a quinidine-like compound, induced, in most experimental settings, similar results as ICI 118.551. Thus, besides its beta-2-adrenoceptor antagonist properties, ICI 118.551 presented other pharmacological actions.
\end{abstract}

Keywords: Langendorff, guinea pigs, ICI 118.551, excitability

Previously in anesthetized intact rats we have shown that the intracerebroventricular (icv) injection of sodium glutamate induced cardiac arrhythmias. We further demonstrated that glutamate induced centrogenic arrhythmias involved a release of acetylcholine from supraspinal structures, followed by a release of norepinephrine and an activation of central beta 1 adrenoceptors. Indeed, selective beta 1 adrenoceptor antagonists, such as practolol, metoprolol, acebutolol prevented

Correspondence should be addressed to

Prof. Barbu Cuparencu

3400 Cluj Napoca 27, Horea Street, Romania 
these arrhythmias, while buthoxamine, a relatively selective beta 2 adrenoceptor antagonist remained inactive $[1,2,3]$.

ICI 118.551 (erythro-DL-1-(7-Methylindan-4-yloxi)-3-isopropylamino-2 butanol hydrochloride) is a selective beta 2 adrenergic blocking agent $[4,5,6]$. The possible therapeutic applications of the compound are restricted to glaucoma [7] and muscular tremor [8].

In anesthetized rats, Berrino et al. [9] demonstrated the lack of effect of ICI 118.551 on the centrogenic cardiac arrhythmias elicited by icv injection of glutamate. However, we noticed in a few experiments, that these beta 2 adrenergic blocking agent could induce centrogenic cardiac arrhythmias, when administered icv alone. These preliminary observations prompted us to study the effects of ICI 118.551 on the cardiac excitability in a more detailed fashion.

We studied the influence of ICI 188.551 on the venticular fibrillation threshold and excitability parameters, in isolated guinea pig hearts. For comparison, the effects of beta 1 adrenergic blocking agent, metoprolol, and a quinidine-like compound, $(+)$ propranolol, were also studied. The actions of these drugs were investigated in hearts from both nontreated and guanethidine pretreated animals. In these experimental setting, guanethidine was used as a monoamine depletor [10].

\section{Materials and methods}

\section{General procedures}

The experiments were carried out in guinea pigs of both sexes, weighing $300-400 \mathrm{~g}$. The animals were sacrificed by decapitation, followed by a rapid exsanguination. The thorax was rapidly opened, the heart removed and connected to the cannula of a Langendorff perfusion device. The time period elapsed between the removal of the heart and the connection to the perfusion device was less than one minute, usually 40 seconds. The capacity of the organ bath was $10 \mathrm{ml}$. The heart was perfused by a Krebs-Henseleit solution at a constant temperature (37 degree Celsius), pressure and $\mathrm{pH}$ (7.4). This later parameter was maintained constant by varying the proportion of $\mathrm{O}_{2} / \mathrm{CO}_{2}$ (approximately $95 \% / 5 \%$ ).

In preliminary experiments, it was shown that during a period of $40 \mathrm{~min}$ the excitability parameters were unchanged. In the experiments reported here, following a 20 min equilibration period, the following parameters were recorded: heart rate, duration of the ventricular monophasic action potential (MAPs), and the cardiac cycle length. MAPs were recorded according to László [11]. The experiments were performed 
with either spontaneously beating or paced hearts. For recording of MAPs pacing or delivery of extrastimuli, silver nonpolarizable needle electrodes were used.

In the paced heart, the heart was paced with a home made stimulator which delivered the minimal current pulses necessary to avoid the occurrence of spontaneous beats, namely a constant train with the following characteristics: $300 \mathrm{~Hz}$ frequency, $1 \mathrm{~ms}$ pulse width, $4-5 \mathrm{~V}$ intensity.

\section{Determination of the "diastolic threshold"}

The diastolic threshold, i.e. the minimal current intensity applied during the late diastole which could elicit a ventricular extrasystole, was determined by using a home made programmable stimulator delivering single pulses of $1.25 \mathrm{~ms}$, variable intensities $(0-100 \mathrm{~mA})$ and constant frequency $(50 \mathrm{~Hz})$. The extrastimuli could be displaced in time during the entire duration of the cardiac cycle by steps of $10 \mathrm{~ms}$ duration.

\section{Determination of the "refractory periods"}

A current of intensity of 2 times the diastolic threshold was used. Extrastimuli were gradually displaced during the cardiac cycle length, starting with $30 \%$ of the MAPs duration until the first ventricular extrasystole occurred. The time of occurrence of this event following the beginning of the MAPs was considered as the "functional refractory period of the ventricle". Then, extrastimuli were delivered at the begining of the MAPs and the corresponding time interval during which no extrasystoles could be induced, was considered as the "effective refractory period of the ventricle" (as reported to the beginning of the MAP).

\section{Determination of "vulnerable period" and "ventricular fibrillation threshold"}

The current intensity was increased 10 times and the delivered pulses were displaced during the functional refractory period, until a ventricular fibrillation ensued. The time interval measured between the beginning of functional refractory period and the appearence of the ventricular fibrillation was termed "vulnerable period". Then the extrastimuli intensity was reduced until no ventricular fibrillation occurred any more. The lowest current intensity which elicited ventricular fibrillation was termed "ventricular fibrillation threshold". 
Determination of "maximal driving frequency"

Finally, the extrastimulus was positioned as described for the determination of the diastolic threshold and the frequency of stimulation was raised until an extrasystole appeared. This was the "maximal driving frequency", a parameter which indirectly indicates the changes in heart excitability $[12,13]$.

\section{Pharmacological procedures}

Following the triplicate determination of these parameters, a 10-minute perfusion of $0.31 \mathrm{mmol} / 1$ of ICI $118.551 ; 0.31 \mathrm{mmol} / 1$ of metoprolol or $0.31 \mathrm{mmol} / 1 \mathrm{l}$ of (+) propranolol (expressed as bases) was started. These doses were chosen following pilot studies for testing the influence of various doses of metoprolol on the parameters of cardiac excitability. The dose of $0.31 \mathrm{mmol} / \mathrm{l}$ of metoprolol was the dose being active in all experiments. Equimolar dose (+) propranolol and ICI 118.551 were then chosen for the present experiments.

During the perfusion period, MAPs were continuously recorded. At the end of the perfusion, the changes in heart excitability were measured as previously described. In addition, the coronary outflow was measured by collecting the fluid into calibrated tubes.

The same procedures were applied in both spontaneously beating heart and paced heart paradigms, either in untreated or guanethidine treated animals. Guanethidine $(25.2 \mathrm{mmol} / \mathrm{kg})$ was injected intraperitoneally 24 hours before the start of the experiment.

\section{Drugs}

The following drugs were used: ICI 118.551 hydrochloride (Zeneca); $\left.{ }^{+}\right)$ propranolol hydrochloride (Zeneca); metoprolol hydrochloride (Ciba-Geigy); guanethidine sulphate (Sicomed, Romania). The doses of all drugs were expressed as bases.

\section{Statistical analysis}

The effect of guanethidine and of each beta-blocker on the studied parameters were analysed by " $\mathrm{t}$ " test. Thus, the unpaired " $\mathrm{t}$ " test was used to compare guanethidine untreated versus guanethidine pretreated groups. The paired "t" test was used for comparison within each group of the pre-versus post drug administration values [14]. Data are expressed as mean $\pm \mathrm{SE}$. Results are considered significant at $\mathrm{p}<0.05$. 


\section{Results}

The effect of each beta-adrenergic blocker on spontaneously beating/paced hearts in animals pretreated or non-pretreated with guanethidine will be separately described.

Effects of ICI 118.551 (Table I)

Spontaneously beating hearts in nonpretreated animals

ICI 118.551 perfusion elicited significant changes in all of the parameters measured. With the exception of the heart rate which decreased (approximately by $25 \%$ ), the rest of the parameters all increased. Note in particular the impressive increase of the diastolic threshold to a value $>100 \mathrm{~mA}$ (increase $>2000 \%$ ) and the ventricular fibrillation threshold to a value $>100 \mathrm{~mA}$ (increase $>70 \%$ ).

The other parameters also increased significantly: the MAPs duration by approximately $35 \%$, the functional and the effective refractory periods by approximately $15 \%$ and $25 \%$, respectively, the vulnerable period by approximately $15 \%$ and the maximal driving frequency by approximately $10 \%$.

Following a $7 \mathrm{~min}$ perfusion, 5-10 isolated ventricular premature beats were noticed. In the following minutes, these rhythm disorders became more and more frequent (up to 25), but they completely disappeared by the end of the perfusion.

\section{Spontaneously beating hearts in guanethidine pretreated animals}

In comparison with the untreated animals, guanethidine injected intraperitoneally 24 hours before the experiments, induced a large increase of the diastolic threshold (approximately by $350 \%$ ) and of the ventricular fibrillation threshold to values $>100 \mathrm{~mA}(>70 \%)$ and also a $20 \%$ increase of the effective refractory period. No extrasystoles were recorded.

Following the perfusion of ICI 118.551, most measured parameters changed significantly. Note a decrease of the diastolic threshold (approximately by 55\%). The vulnerable period duration and ventricular fibrillation threshold $(>100 \mathrm{~mA})$ were not determinable in both pre- and post/- ICI 118.551 perfusion.

We also noticed a significant bradycardia (approximately by $15 \%$ ), an increase of the MAPs duration by $20 \%$, an increase of the functional and effective refractory periods (approximately by $20 \%$ and $10 \%$, respectively) and a decrease of the maximal driving frequency (approximately by $10 \%$ ).

In only one experiment, did ICI 118.551 elicit a single extrasystole. 


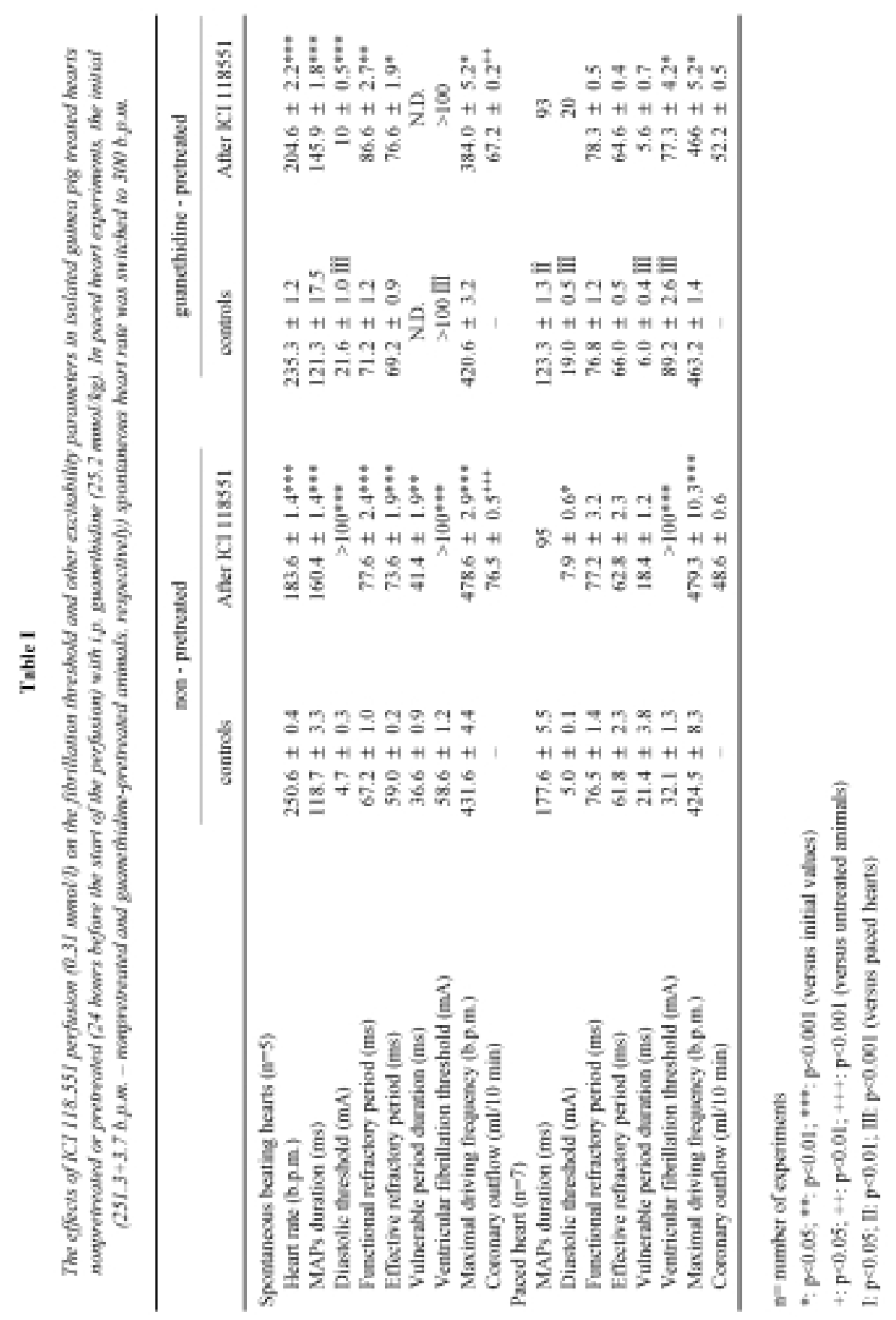




\section{Paced hearts in nonpretreated animals}

Note the $60 \%$ increase of the diastolic threshold, the impressive augmentation to a value higher than $100 \mathrm{~mA}$ of the ventricular fibrillation threshold (increase $>200 \%$ ) and the increase of $10 \%$ of the maximal driving frequency.

It is noteworthy that during the perfusion period, a few ventricular extrasystoles (3-5) occurred. We also observed decrease the coronary outflow (approximately by $60 \%$ ) in paced hearts compared to spontaneously beating hearts.

\section{Paced hearts in guanethidine pretreated animals}

Compared to the untreated animals, guanethidine injected intraperitoneally 24 hours before the experiments, induced the following important changes in the control animal: increase of the diastolic threshold (approximately by $280 \%$ ), and increase of the ventricular fibrillation threshold (approximately by $180 \%$ ). Note also the decrease of the MAPs duration (approximately by $30 \%$ ) and decrease of the duration of the vulnerable period (approximately by $70 \%$ ).

Following the perfusion of ICI 118.551 in guanethidine treated animals, we observed a significant decrease of the ventricular fibrillation threshold (approximately by $15 \%$ ) and a significant decrease of the maximal driving frequency (approximately by $10 \%)$.

Note the decrease of the coronary outflow (approximately by 20\%) in paced hearts compared to spontaneously beating hearts. No other changes occurred.

Effects of metoprolol (Table II)

\section{Spontaneously beating hearts in nonpretreated animals}

The perfusion of metoprolol induced a very significant increase of the functional and effective refractory periods (approximately by $30 \%$ and $60 \%$, respectively) and a very significant elevation of the ventricular fibrillation threshold (approximately by $80 \%$ ). Note also the significant bradycardia (approximately by $20 \%$ ), and the increase of the MAPs duration (approximately by $10 \%$ ).

Metoprolol perfusion elicited a progressive increase of the number of premature beats of ventricular origin (initially $3-5$, finally $10-15$ ). They appeared with a $2 \mathrm{~min}$ onset and drastically diminished at the end of perfusion. 


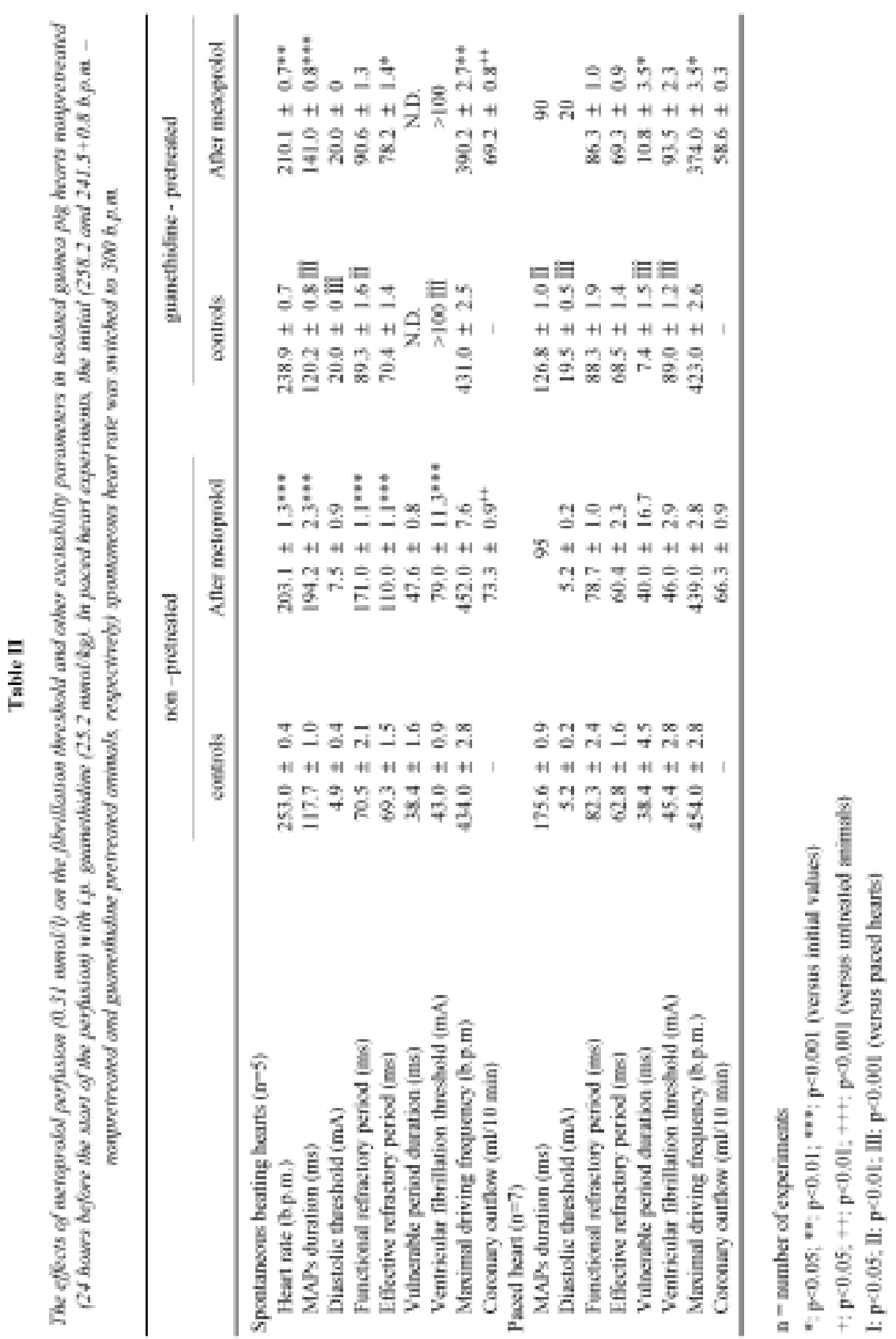




\section{Spontaneously beating hearts in guanethidine treated animals}

In comparison to the untreated control animals, guanethidine induced the following changes: increase of $170 \%$ of the diastolic threshold and significant rise of the fibrillation threshold to values $>100 \mathrm{~mA}$ (increase of $>130 \%$ ). Note that the vulnerable period duration was not determinable. Other changes consisted in significant decreases of the MAPs duration (approximately by $30 \%$ ) and functional refractory period (approximately by $40 \%$ ).

Before or following the perfusion of metoprolol, the ventricular fibrillation threshold was so high that it could not be measured ( $>100 \mathrm{~mA})$.

Following metoprolol perfusion, we also detected a significant bradycardia (approximately by $15 \%$ ), a significant increase of the Maps duration (approximately by $20 \%$ ) and the effective refractory period (approximately by $10 \%$ ) and a decrease of the maximal driving frequency (approximately by $10 \%$ ).

In guanethidine pretreated hearts, premature beats (2-5) were also observed.

\section{Paced hearts in nonpretreated animals}

No significant changes of the investigated parameters occurred. During the metoprolol perfusion, some ventricular extrasystoles (2-4) were noticed. As expected, the coronary outflow was lower for these paced hearts, as compared to the spontaneously beating hearts (approximately by $10 \%$ ).

\section{Paced hearts in guanethidine pretreated animals}

Compared to the untreated control animals, guanethidine induced the following changes: an increase of the diastolic threshold (approximately by 27\%) and an increase of the ventricular fibrillation threshold (approximately by $90 \%$ ). Note the decrease of the MAPs duration (approximately by $30 \%$ ) and the vulnerable period duration (approximately by $80 \%$ ).

Following the perfusion of the metoprolol in these pretreated animals, a significant increase of the vulnerable period (approximately by $45 \%$ ) and a significant decrease of the maximal driving frequency (approximately by $10 \%$ ) occurred.

Effects (+) of propranolol (Table III) 


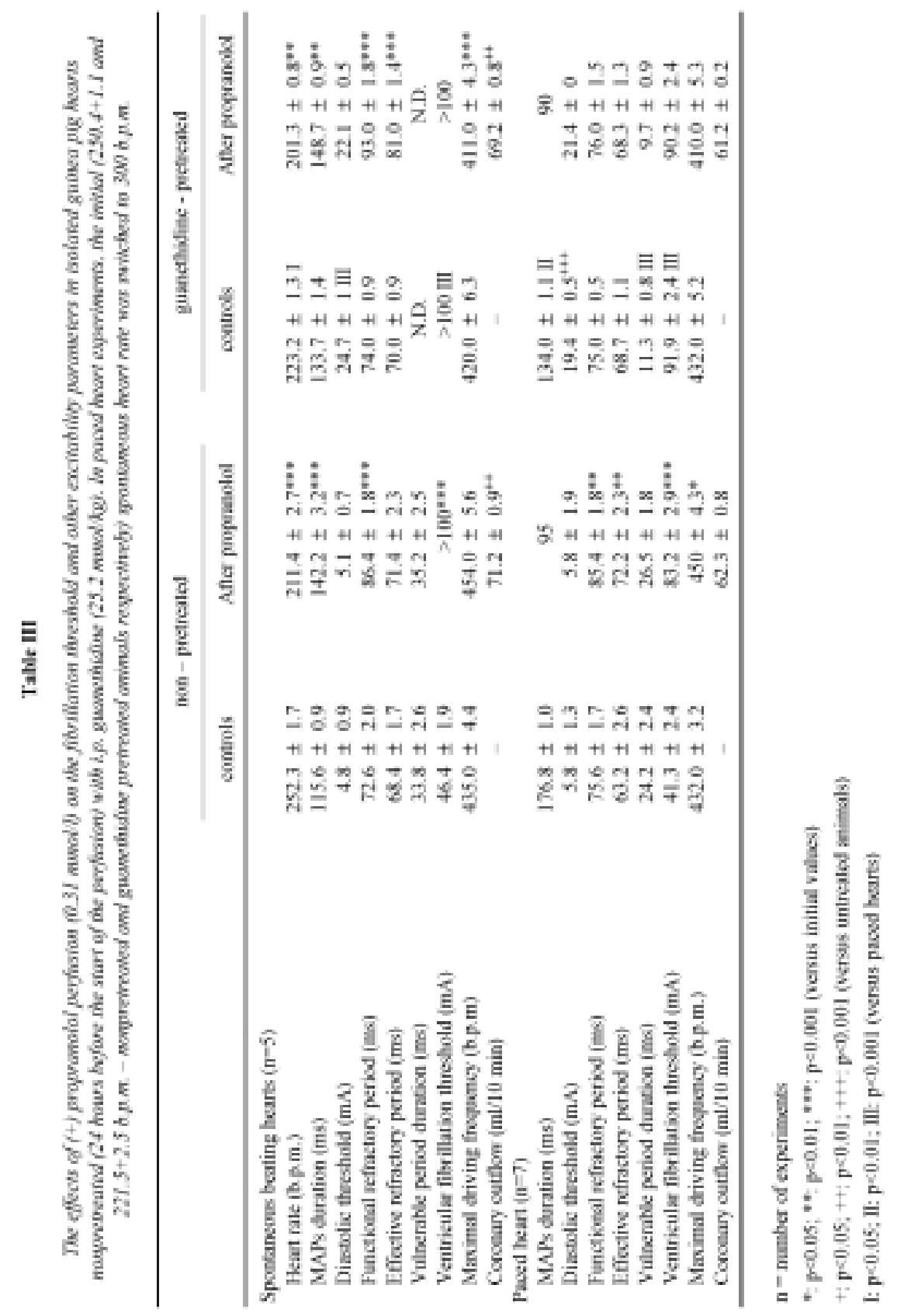




\section{Spontaneously beating hearts in nonpretreated animals}

(+) Propranolol perfusion induced an important increase of the ventricular fibrillation threshold (approximately by $110 \%$ ). A reduction of the heart rate (approximately by $15 \%$ ) and an increase of the functional refractory period (approximately by $20 \%$ ) were also noted. Only some isolated extrasystoles (1-3) were detected during the perfusion. They disappeared soon after the end of perfusion.

\section{Spontaneously beating in guanethidine pretreated animals}

As compared to the untreated control animals, guanethidine induced the following changes: an increase of the diastolic threshold (approximately by 400\%) and a significant rise of the fibrillation threshold to values $>100 \mathrm{~mA}$ (increase of $>100 \%$ ). Note the bradycardia (approximately by $10 \%$ ) and the fact that vulnerable period duration was non determinable; no extrasystoles were recorded.

Either pre- or post $(+)$ propranolol perfusion, the vulnerable period and the ventricular fibrillation threshold $(>100 \mathrm{~mA})$ could not be measured, being too high for the possibilities of our device to measure it.

Following the perfusion of $(+)$ propranolol, we also detected the following changes: bradycardia (approximately by $10 \%$ ), increase of the MAPs duration by $25 \%$ and $15 \%$ for the functional and effective refractory periods, respectively).

\section{Paced hearts in nonpretreated animals}

Note a significant increase of the ventricular fibrillation threshold of approximately $100 \%$. Other changes consisted in an increase of the functional and effective refractory periods (approximately by $10 \%$, respectively $15 \%$ ) and of the maximal driving frequency (approximately by $5 \%$ ).

During the perfusion, a relatively large number of ventricular extrasystoles (10-17) was also noticed. As already described, the coronary outflow was lower for these paced hearts, as compared to the spontaneously beating hearts (approximately by $10 \%$ ).

\section{Paced hearts in guanethidine treated animals}

As compared to the untreated control animals, guanethidine induced changes similar to those already described, namely a very significant increase (approximately by $200 \%$ of the ventricular fibrillation threshold). Other changes consisted in a decrease of the MAPs duration of approximately by $25 \%$ and the vulnerable period duration of $50 \%$. 
The perfusion of $(+)$ propranolol in these pretreated animals failed to affect the investigated parameters. In these paced hearts the extrasystoles [15-20] failed to disappear after the end of perfusion.

\section{Discussion}

During the perfusion of the ICI 118.551, premature ventricular beats were frequently seen. It is noteworthy that the extrasystoles appeared not only under the influence of ICI 118.551 perfusion, but also when two well-known antiarrhythmic drugs metoprolol and $(+)$ propranolol were perfused. These findings enabled us to think that there is a nonspecific phenomenon, caused by the contact of foreign substances with the heart. This effect is transient (this is an argument that the extrasystoles are of a nonspecific character). Since a similar effect is not visible in guanethidine treated animals, it may be concluded that it is due to a release of catecholamines in an active form.

A surprising statement was that similar effects were seen with metoprolol and (+) propranolol. Since guanethidine preadministration failed to affect the occurrence of arrhythmias in these later cases, we suggest that they were probably due to mechanisms differing from those invoved in the effect of ICI 118.551. Since both drugs have a quinidine-like action [16] and since these types of drugs, when given in high doses, frequently induce arrhythmias by a re-entry mechanism, it is tempting to ascribe the extrasystoles seen during the perfusion period to this arrhytmogenic mechanism.

ICI 118.551 also induced a significant decrease of the heart rate both in controls and guanethidine treated animals. This conclusion contrasts with the data of Bilski et al. [5], who stated that in both controls and syrosingopine treated rats, ICI 118.551 failed to induce any changes in the heart rate. This could be due to different experimental conditions (different species, in vitro vs. in vivo experiments).

It is noteworthy that ICI 118.551 also exhibits a local anaesthetic and membrane stabilising activity. It is not clear whether this property would contribute to the antiarrhythmic effects of ICI 118.551 in coronary ligated rats [5].

All drugs investigated, namely metoprolol, $(+)$ propranolol and ICI 118.551 elicited an increase of the ventricular fibrillation threshold in guanethidine pretreated animals, as revealed by the experiments on paced hearts. This later effect of ICI 118.551 seems to be due to a depletion of some endogenous substance stores following guanethidine pretreatment. The substances depleted by guanethidine, with an influence of the profibrillatory effect of ICI 118.551 were certainly not catecholamines, since these compounds possess essentially a threshold-lowering activity [13-17]. Another putative candidate is serotonin which is also depleted by guanethidine. But serotonin 
has also a fibrillation threshold reducing activity as shown by Szekeres and Papp [13] and Cuparencu et al. (unpublished data). It is not excluded that the profibrillatory activity of ICI 118.551 was unmasked following guanethidine pretreatment by depletion of acetylcholine and gamma-aminobutyric acid (GABA) [10], exerting a contrary action in intact animals.

It has to be mentioned that the spontaneously beating heart, the perfusion of ICI 118.551, besides the increases of the ventricular fibrillation threshold, also elicited an increase of the refractory periods. However, there is probably no link between the changes in the refractory periods and the changes in the fibrillation threshold since no changes of the refractory periods were noticed in paced hearts, while the fibrillation threshold could be modified. Other authors $[5,18]$ also noticed no link between changes in cardiac excitability and those in fibrillation threshold. The fibrillation threshold measures more the electrical inhomogeneity of the heart than its excitability. Thus, in certain conditions, these parameters may change independently.

Metoprolol induced a very significant increase of the ventricular fibrillation threshold, but only in spontaneously beating hearts. Note that metoprolol did not induce a decrease of the fibrillation threshold in guanethidine pretreated animals, as did ICI 118.551 , but rather a slight increase. The increase of the ventricular fibrillation threshold could be secondary to the decrease of the animals heart rate [13]. Tosaki et al. [19] also demonstrated that metoprolol exhibited an antifibrillatory effect, related to its negative chronotropic activity. In spontaneously beating hearts, the ventricular fibrillation threshold increased in parallel with the refractory periods and fibrillation threshold. Guanethidine pretreatment failed to have any influence in paced hearts and increased the bradycardic effects of $(+)$ propranolol (in spontaneously beating hearts).

Metoprolol and $(+)$ propranolol elicited ventricular extrasystoles, and this effect was not influenced by guanethidine pretreatment. An exception was the absence of extrasystoles in $(+)$ propranolol perfused spontaneously beating hearts. Metoprolol and $(+)$ propranolol perfusion induced a very significant increase in the ventricular fibrillation threshold, not influenced by guanethidine pretreatment. In spontaneously beating hearts, the fibrillation threshold increases were accompanied by elevations of the refractory periods.

\section{Acknowledgements}

The authors thank professor G. Cheymol (Paris, France) for advice in the preparation of the manuscript. They are greatly indebted to Zeneca (Alderley Park, Cheshire, U.K.) for the generous gift of ICI 118.551 and (+) propranolol and to Ciba-Geigy (Basel, Switzerland) for metoprolol. 


\section{REFERENCES}

1. Cuparencu, B.: Arrhythmogenic and antiarrhythmic effects of some centrally administered drugs in rats. In: Cardiovascular Pharmacology '87, ed Papp, J. Gy., Akadémiai Kiadó, Budapest 1987, p. 137-144.

2. Cuparencu, B.: Effects of cardiovascular drugs mediated through the central nervous system (in Romanian), Editura medicala, Bucharest 1988.

3. Cuparencu, B., Safta, L., Papilian, V. V., Sandor, V. I., Fabian, A., Horak, J.: Cholinergic-adrenergic interactions in the genesis of centrogenic arrhythmias in the rats. In: Neuropharmacology '85, eds Kelemen, K., Magyar, K., Vizi, Sz. Akadémiai Kiadó, Budapest 1985, pp. 209-217.

4. O’Donnel, S. R., Wanstall, J. C.: Evidence that ICI 118.551 is a potent, highly beta 2-selective adrenoceptor antagonist and can be used to characterize beta adrenoceptor populations in tissues. Life Sci. 27, 671-677 (1980)

5. Bilski, A. J., Haliday, S. E., Fitzgerald, J. D., Wale, J. L.: The pharmacology of beta 2-selective adrenoceptor antagonist (ICI 118.551) J. Cardiovasc Pharmacol. 5, 430-437 (1983).

6. Rimele, T. J., Henry, D. E., Giesa, F. R., Buckley, S. K., Geiger, G., Heaslip, R. J., Lee, D. K. H., Grimes, D.: Comparison or the beta-adrenoceptor affinity and selectivity of cetamolol, atenolol, betaxolol and ICI 118.551. J. Cardiovasc. Pharmacol. 12, 208-217 (1988).

7. Nathason, J. A.: ICI 118.551: An effective ocular hypotensive agent with minimal cardiac side-effects. Br. J. Pharmacol. 83, 821-829 (1984).

8. McCaffrey, P. M., Riddell, J. G., Arnold, J. M. O., Nelson, J. K., McDevitt, D. G.: Comparison of the effects of propranolol, metoprolol and ICI 118.551 on the CVS and finger tremor in hyperthyroidism. Br. J. Pharmacol. 26, 675 (1988).

9. Berrino, L., Cuparencu, B., Safta, L., Stella, L., Sandor, V. I., De Santis, D., Arustei, V., Marrazo, R., Maione, S., Carnevale, M., Gozariu-Strimbu, M., Marmo, M.: Do the central beta 2-adrenoceptor play any role in the genesis of centrogenic cardiac arrhythmias in anesthetized rats? Curr. Ther. Res. 49, 282-289 (1991).

10. Marmo, E.: Farmacologia generale e speciale, Turin: Utet 1991, pp. 617-633.

11. László, J.: Problems of the biopotentials recording methods. The decrease of the shunt (in Hungarian), Rev. Med. (Tg. Mures) 27, 144-151 (1981).

12. Dawes, Gs.: Experimental cardiac arrhythmias and quinidine-like drugs. Pharmacol. Rev. 4, 43-84 (1952).

13. Szekeres, L., Papp, J. G.: Experimental cardiac arrhythias and antiarrhythmic drugs. Akadémiai Kiadó, Budapest 1971, pp. 58-64, and pp. 80-81.

14. Snedecor, G. W., Cochran, W. G.: Statistical methods. VIth ed, Iowa Univ. Press Ames, Iowa, 1978, p. 91.

15. Winslow, E., Walke, R. G., Mason, R.: Antiarrhythmic effects of selective beta 1- and nonselective beta-adrenoceptor blokade in normokalaemic and dietary-induced hypokalaemic rats. J. Cardiovasc. Pharmacol. 14, 673-680 (1989).

16. Lucchesi, B.: Antiarrhythmic drugs. In: Cardiovascular Pharmacology. IIIrd edition, Antonaccio, M. J., New-York Raven Press, 1990, pp. 369-483.

17. Marmo, E.: Farmacoterapia delle aritmie cardiache. 2nd ed., Minerva Medica, Turin 1986.

18. Antoni, H: What is measured by the electrical threshold for fibrillation? In: Cardiovascular Pharmacology '87. ed Papp, J. Gy., Akadémiai Kiadó, Budapest 1987, pp. 31-44.

19. Tosaki, A., Szekeres, L., Pearse, D. J.: Metorpolol reduces reprefusion-induced fibrillation in the isolated rat heart: protection is secondary to bradycardia. J. Cardiovasv. Pharmacol. 10, 489-497 (1987). 